\title{
Appel de candidatures : Prix de recherche et d'enseignement de l'ACG et membres aux comités de l'ACG
}

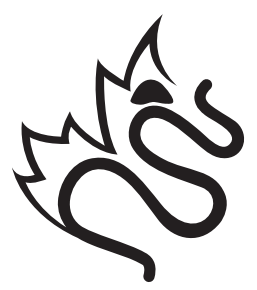

L'Association Canadienne de Gastroentérologie

For English see page 640

\begin{abstract}
APPEL DE CANDIDATURES - PRIX DE RECHERCHE ET D'ENSEIGNEMENT DE L'ACG

Les membres de l'Association canadienne de gastroentérologie (ACG) sont invités à proposer des candidatures aux prix de recherche et d'enseignement 2009 de l'ACG. Tous les candidats doivent être membres de l'ACG. La description des prix, les critères d'admissibilité et le processus de candidature figurent ci-dessous :
\end{abstract}

Prix de recherche

Professeur visiteur en recherche de l'ACG : Offert chaque année (mais pas nécessairement attribué) à un enseignant démontré et un chercheur de premier plan. Le professeur visiteur se rend dans plusieurs centres du Canada pour donner une présentation dans le cadre d'une séance scientifique en gastroentérologie ou en médecine générale ou de séminaires de recherche.

Prix d'excellence de l'ACG en recherche : Chaque année, le comité de recherche sélectionne un chercheur canadien remarquable qui présentera la conférence annuelle sur la recherche lors de la Semaine canadienne sur les maladies digestives (SCMD).

Prix du jeune chercheur de l'ACG : Ce prix rend hommage à un apport exceptionnel à la gastroentérologie par des recherches fondamentales ou cliniques menées par un jeune chercheur invité à faire une conférence lors de la SCMD. Les candidats doivent :

- avoir moins de 45 ans et détenir leur premier poste universitaire depuis moins de sept ans;

- détenir un poste dans une université, un hôpital ou un institut de recherche du Canada;

- être citoyens canadiens ou résidents permanents du Canada.

Prix d'enseignement

Prix d'excellence de l'ACG en enseignement : Ce prix est offert chaque année pour un apport remarquable à l'enseignement sur la scène nationale ou internationale, dans les domaines de l'enseignement, de la recherche en enseignement ou de l'élaboration de programmes d'enseignement. Le lauréat est invité à donner une conférence lors de la SCMD.

Prix du jeune formateur de l'ACG : Ce prix salue un apport remarquable à l'enseignement de la gastroentérologie de la part d'un jeune chercheur, qui est invité à donner une conférence lors de la SCMD. Les candidats doivent :

- avoir moins de 45 ans et détenir leur premier poste universitaire depuis moins de sept ans;

- détenir un poste dans une université, un hôpital ou un institut de recherche du Canada;

- être citoyens canadiens ou résidents permanents du Canada.

Processus de candidature

Pour proposer des candidatures aux prix précédents, les membres de l'ACG peuvent remplir le formulaire pdf par voie électronique, dans le site de l'ACG (dans la zone Research, CAG Research Awards ou Physician Education, Awards), à l'adresse <www.cag-acg.org/uploads/cagyinominationformweb.pdf>, ou télécharger le formulaire et le télécopier ou le poster au bureau national de l'ACG après l'avoir rempli :

Bureau national de l'ACG 2902 South Sheridan Way, bureau 201

Oakville (Ontario) L6J 7L6

Télécopieur : 905-829-0242

Courriel : cagoffice@cag-acg.org

Un exemplaire du curriculum vitæ du candidat et une lettre d'appui doivent accompagner toutes les candidatures.

Les comités de la recherche et de l'enseignement examineront les candidatures et proposeront des recommandations au comité directeur qui prendra la décision définitive au sujet des lauréats. La sélection sera fondée sur le dossier de recherche ou de formation des candidats.

L'échéance des candidatures est fixée au 31 juillet 2008.

L'ACG est fière de présenter ses bienfaiteurs :
Abbott Canada
AstraZeneca Canada Inc
Axcan Pharma Inc
Nycomed Canada Inc
Olympus Canada Inc
Pentax Canada Inc
Procter \& Gamble Pharmaceuticals
Schering Canada Inc 


\section{APPEL DE CANDIDATURES - MEMBRES AUX COMITÉS DE L'ACG}

Les membres de l'ACG sont invités à s'engager au sein de l'organisme pour contribuer à promouvoir son développement et la gastroentérologie au Canada. Le comité de mises en candidature est à la recherche de membres intéressés à siéger à un comité de l'ACG. Les mandats sont de trois ans, et les comités «se réunissent » par téléconférences périodiques. La fréquence de ces téléconférences est déterminée par les activités de chaque comité. Les comités qui ont besoin de représentants pour le mandat 2009 à 2012 sont énumérés cidessous, conjointement avec une brève description de chacun d'eux.

\section{Les comités de l'ACG}

Archives : les dossiers et l'historique de l'ACG.

Formation : Promouvoir et diffuser les documents d'enseignement et de formation pour l'ACG et orienter les divers comités d'enseignement.

Endoscopie : Représenter l'ACG à l'égard de questions reliées aux endoscopistes et promouvoir la compétence et la formation en endoscopie.

Finances : Conjointement avec le bureau national et le comité directeur, établir des stratégies de collecte de fonds et les implanter pour obtenir des commandites d'entreprise et pour tenir des initiatives clés comme la SCMD et les projets de recherche.

Pédiatrie : Représenter les intérêts des gastroentérologues pédiatres auprès du conseil d'administration et des membres.

Questions de pratique : Traiter de questions pertinentes pour l'exercice de la gastroentérologie. Des représentants de
l'Alberta, de l'Ontario et des Maritimes sont recherchés pour 2009 à 2012.

Recherche : Promouvoir la recherche fondamentale et la recherche clinique, comme suit :

- examiner et sélectionner les candidats qui recevront les subventions de recherche;

- examiner et choisir les résumés pour la SCMD et les prix aux étudiants;

- participer à l'élaboration du programme de la SCMD.

Si vous êtes intéressé à siéger à un comité de l'ACG ou si vous connaissez un membre qui le serait, vous êtes invité à soumettre des candidatures au bureau national de l'ACG par la poste, par télécopieur ou par courriel :

Bureau national de l'ACG

2902 South Sheridan Way, bureau 201

Oakville (Ontario) L6J 7L6

Télécopieur : 905-829-0242

Courriel : cagoffice@cag-acg.org

Les candidatures seront examinées par le comité de mises en candidature, qui proposera des candidats au comité de gouvernance afin d'obtenir leur approbation. Les candidatures approuvées par le comité de gouvernance seront présentées aux membres et recevront l'approbation définitive à l'assemblée générale annuelle.

L'échéance des candidatures est fixée au 31 août 2008. 


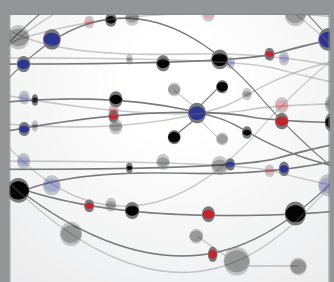

The Scientific World Journal
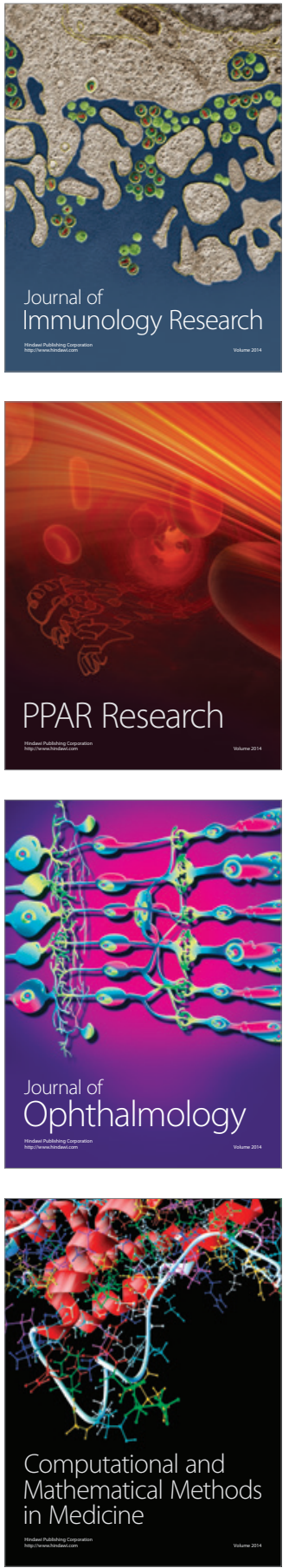

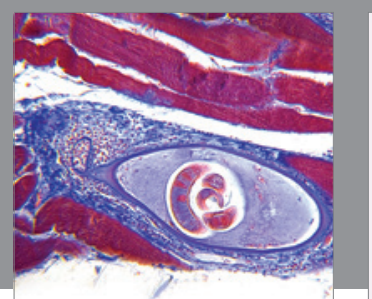

Gastroenterology Research and Practice

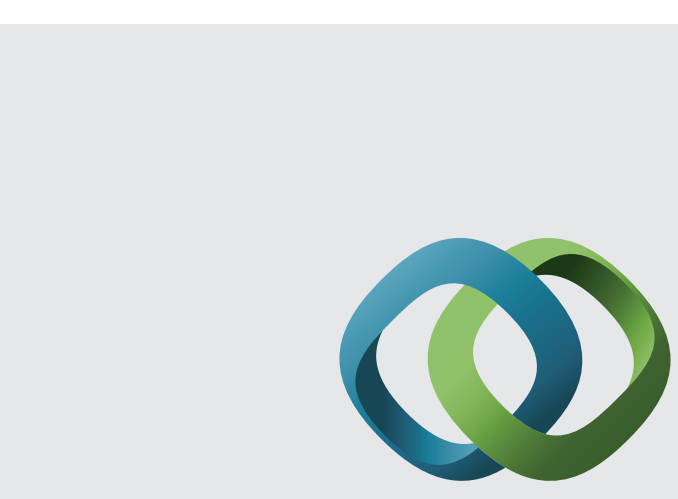

\section{Hindawi}

Submit your manuscripts at

http://www.hindawi.com
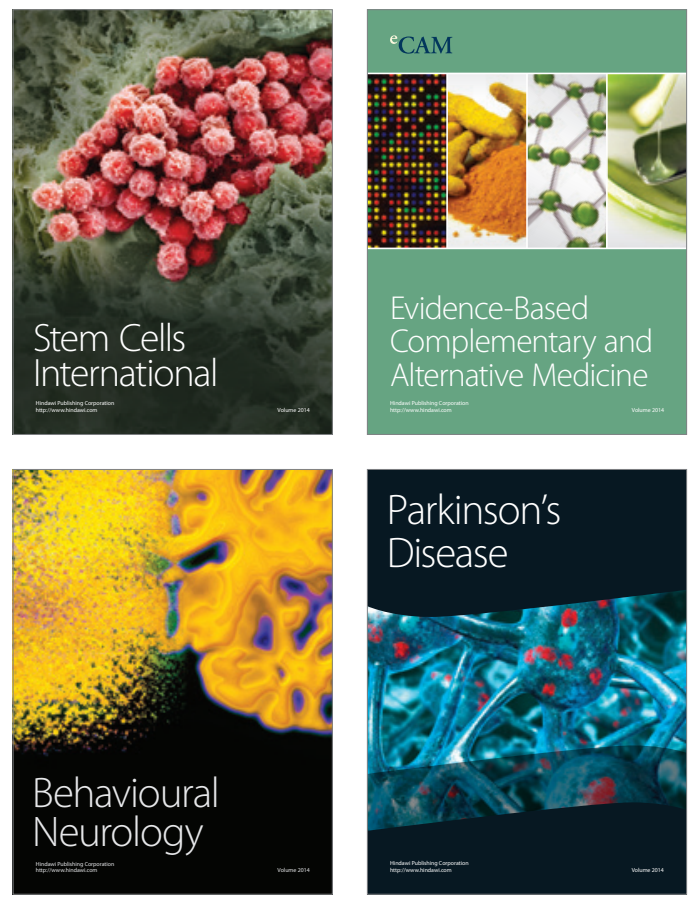
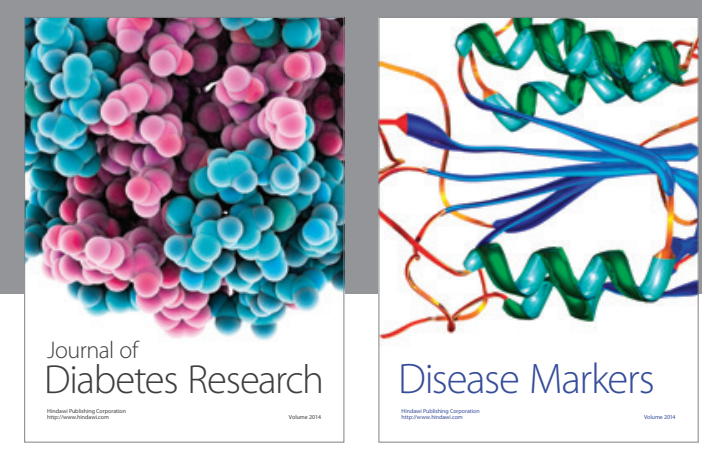

Disease Markers
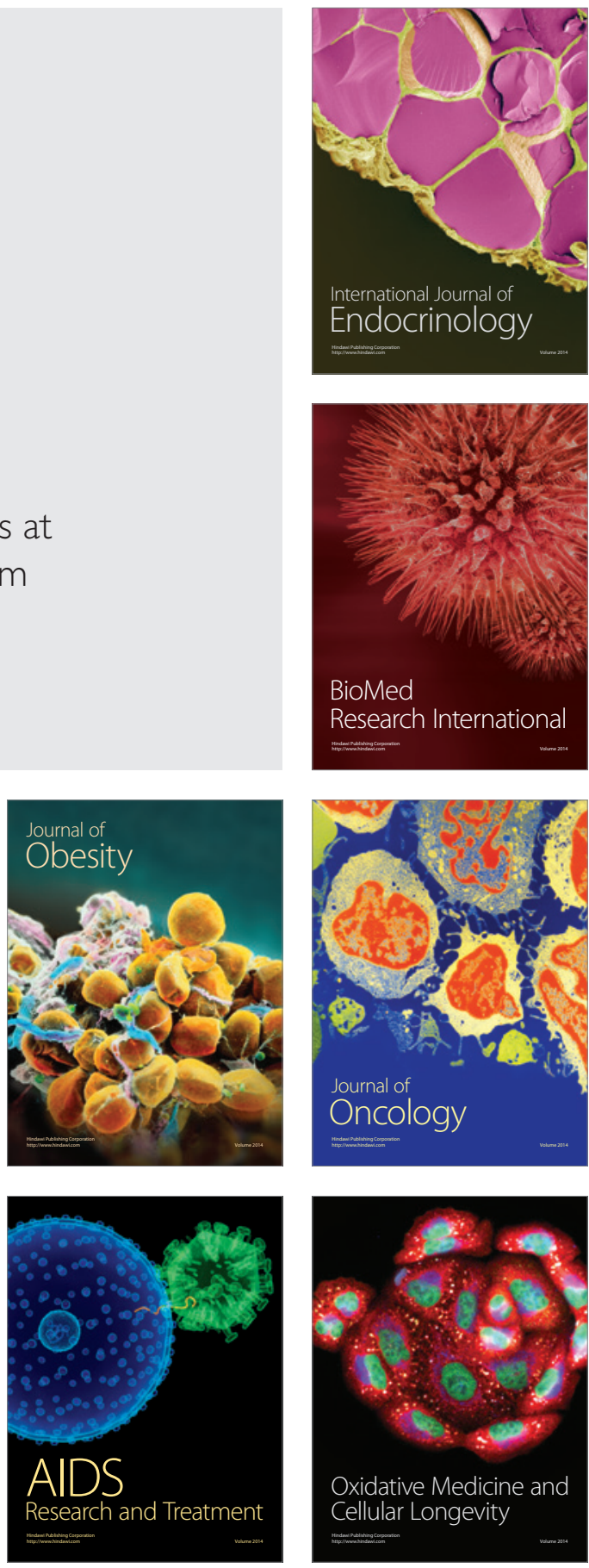